\title{
Phantom Breast Syndrome: Experience from a University Hospital in a Developing Country
}

\author{
Fatimah Al Qatari, Noor Al Herz and Maha Abdel Hadi* \\ Department of surgery, Imam Abdulrahman Bin Faisal University, Dammam -Saudi Arabia
}

*Corresponding author: Maha Abdel Hadi, Breast Division, Department of Surgery, Imam Abdulrahman Bin Faisal University. Saudi Arabia

\begin{abstract}
Aim: The aim of this study to assess the presence and the significance of phantom breast syndrome (PBS) in a conservative community with the cultural stigma of emotion disclosure related to mastectomy.

Materials and Methods: This study was conducted at the breast oncology clinics between Jan 2017-December 2018. Direct interview using a prepared pilot tested structured questionnaire directed towards patients who underwent mastectomy between the periods of January 2011-December 2017. Pain and body image distortion were the focus of the study. Demographic data, marital status, education and socioeconomic status were included. The target population intended was 100 patients.

Results: 347 patients were diagnosed with breast cancer, mastectomy was performed on 143 (41.2\%). The capture rate for interview was $82(82 \%)$ of the targeted interviewees. Age ranged between 23-83years with the median age of 49 years. The majority of women who experienced phantom breast syndrome $30(36.5 \%)$ were within the reproductive age group. Progression of pain after the first year of mastectomy was noticeable ranging from phantom pain to severe pain requiring aid with standard analgesics with successful relief in $57(69.5 \%)$ of the patients. Body image distortion concerns and phantom pain symptoms were more pronounced in married 29 (35\%), highly educated 32 (39\%) and high socioeconomic class 13 (16\%) compared to the rest of the group. It was also noticed that pain symptoms and severity decrease with age.
\end{abstract}

Conclusion: PBS remains a significant entity that should be identified, investigated and treated in order to contribute to high quality of life in these subsets of women with breast cancer. Special tailoring to own cultures may aid in rehabilitation of the affected women.

\section{Introduction}

Mastectomy remains the treatment of choice in many developing countries due factors influencing the early detection strategies. Post mastectomy pains is often masked by the feeling of inadequacy and shame of the mutilated body image. Phantom pain is the pain that represent the feeling of the pain of an amputated body part. With Phantom Breast Syndrome" (PBS) patients may suffer the sensation which varies from mild discomfort itching, tingling, pressure, burning, and throbbing at the target site. The timing of the symptoms is variable ranging from few months to years [1]. In addition to the mutilated body image, it is estimated that over $50 \%$ of women suffer chronic pain following treatment for breast cancer surgery. " [2] It can seriously affect quality of life through the combined impact of physical disability and emotional distress. [3] Some reports have shown that genetic variations in the catecholaminergic and serotonergic pathways may contribute to the development and severity of persistent breast pain [4]. The severity and impact of PBS varies among individuals, it may be affected by age, education, axillary dissection, reconstruction, and psychosocial stress [5]. In our communities, pain may not be the major concern, women present late due to the fear of mutilating mastectomy and the social stigma it accompanies. Another entity is Alexithymia, or the inability to express emotions maybe involved in mechanisms of comprehending pain chronicity [6].

\section{Materials \& Methods}

This study was conducted at the breast oncology clinics between Jan 2017-December 2018. Direct interview using a prepared pilot tested structured questionnaire directed towards patients who underwent mastectomy between the periods of January 
2011-December 2017. Pain and body image distortion were the focus of the study. Demographic data, marital status, education and socioeconomic status were included. The target number intended was 100 patients. Statistical analysis was performed using SPSS version 20.

\section{Results}

The total number of patients diagnosed with breast cancer for the above period was 347, mastectomy was performed on 143 (41.2\%). According to the readiness of patients and the logistics available $82(57 \%)$ of the mastectomy group were interviewed. Age ranged between 23-83years with the median age of 49 years. 47 (57\%) were married. Pain in patients progressed after the first year of mastectomy ranging from phantom symptoms to severe pain requiring aid with standard analgesics leading to successful relief in 57 (69.5\%) It was also noticed that pain symptoms and severity decrease with age (Figure 1).The majority of women who experienced phantom breast syndrome 30 (36\%) were within the reproductive age group. Body image distortion concerns and phantom pain symptoms were the highest 29 (35\%) in the married patients as compared to the rest of the group. This may be influenced by the psychosocial effect and attempts of women to portray a perfect body image. Education and socioeconomic statuses were other parameters used to assess patients' concerns. It was noticed that both high education 32 (39\%) and high socioeconomic status 13 (16\%) statuses were important factors in inducing more concerns with body image distortion than the less educated lower socioeconomic group. Again, this result may reflect the leading role of these women in the community.

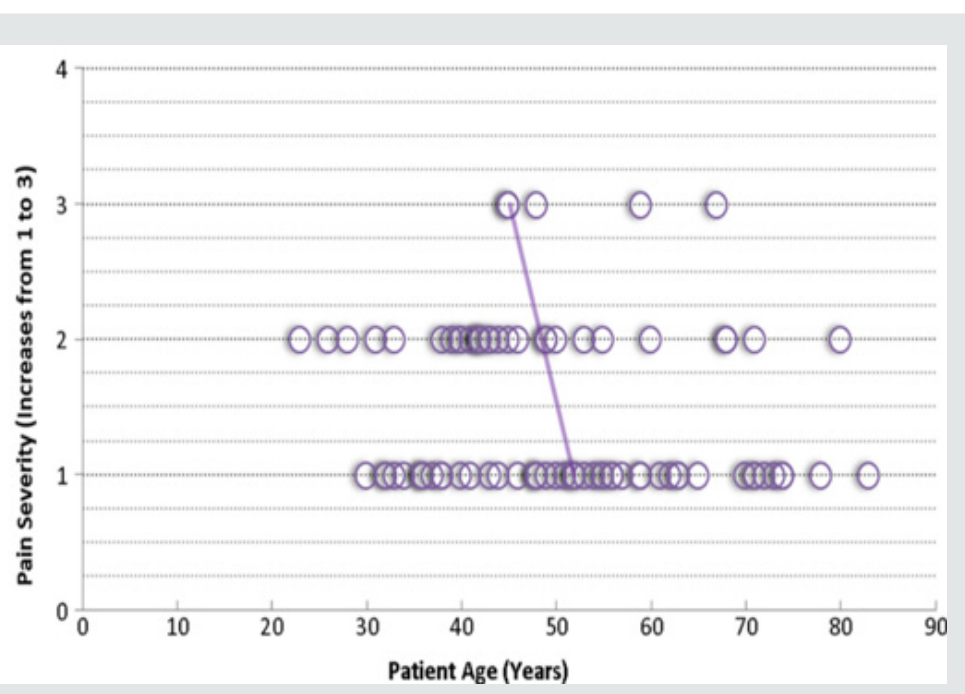

Figure 1: Illustrates the reduction of pain symptoms with age.

\section{Discussion}

PBS is an increasingly recognized as a significant public health problem. Chronic and persistent PBS is a common condition, its potential severity has dictated special attention to understanding and treating this entity [7]. Despite advances treatment of breast cancer, cancer pain remains an under assessed and undertreated as this group exhibit more tolerance to pain. Many reports have shown that younger patients react differently in the form of different avoidance-related and relational challenges [8] Anatomic site characterization of pain is not well delineated in most women. Studies have suggested separate evaluations to identify persistent pain conditions. This pain is further complicated in patients receiving treatment for palliative care where they experience variety of pain related to other concurring symptoms. Patients with high symptoms were seen in younger patients with poor quality of life scores [9].

In our culture driven societies, the stigma of breastcancer disease is a major dilemma. Marital status, education and socioeconomic statuses play a major role on perception of symptoms. In this study being married, highly educated and from high socioeconomic classes tend to express relatively more concern for body image distortion than pain. Lower education, lower socioeconomic statuses have different interests and concerns. Their main apprehension was pain, physical appearance is perceived as luxury, thus, the physical image concerns is less pronounced in in this group this observation seems to be driven by the shame coupled by the peer pressure exerted by ones' community. Many emerging studies suggest close association between persistent PBS and psychosocial regardless of the surgical and medical treatment that patients receive may lead to new strategies in rehabilitation [10,11]. Immune mechanisms may also play a role in the development and maintenance of persistent pain related to variations in cytokine genes and genotyping array. In addition, cancer treatment may influence the appearance of variety of symptoms $[9,12,13]$. Extensive researches are now focused on social, psychological, and functional sequelae of this syndrome with emphasis on current pharmacological, psychological, and rehabilitative measures to aid in successful approaches to treatment. $[14,15]$ 


\section{Conclusion}

PBS remains a significant entity that should be identified, investigated and treated in order to contribute to high quality of life in these subsets of women with breast cancer. Special tailoring to own cultures may aid in rehabilitation of the affected women.

\section{References}

1. Ramesh, Shukla N, Bhatnagar S (2009) Phantom Breast Syndrome Indian J Palliat Care. Jul-Dec 15(2): 103-107.

2. Staps T, Hoogenhout J, Wobbes T (1985) Phantom breast sensations following mastectomy. Cancer 56(12): 2898-2901.

3. Kroner K, Knudsen UB, Lundby L, Hvid H (1992) Long - term phantom breast syndrome after mastectomy. Clin J Pain 8(4): 346-350.

4. Mitchell R Knisely, Yvette P Conley, Kord M Kober, Betty Smoot, Steven M Paul, et al. (2018) Associations Between Catecholaminergic and Serotonergic Genes and Persistent Breast Pain Phenotypes After Breast Cancer Surgery. The Journal of Pain 19(10): 1130-1146.

5. Kristin L Schreiber, Nantthasorn Zinboonyahgoon, Xinling Xu, Tara Spivey Tari King, et al. (2018) Preoperative Psychosocial and Psychophysical Phenotypes as Predictors of Acute Pain Outcomes After Breast Surgery S1526-5900(18): 30897-30906.

6. Sophie Baudic, Christian Jayr, Aline Albi Feldzer, Jacques Fermanian, Anne Masselin Dubois, et al. Nadine Attal Effect of Alexithymia and Emotional Repression on Postsurgical Pain in Women With Breast Cancer: A Prospective Longitudinal 12-Month Study. The Journal of Pain 17(1): 90-100.

7. Tait R, Zoberi K, Ferguson M, Levenhagen K, Luebbert R, et al. (2018) Persistent Post-Mastectomy Pain: Risk Factors and Current Approaches to Treatment. The Journal of Pain 19(12) : 1367-1383.
8. Mah K, Tran T, Gauthier L, Rodin G, Zimmermann C, et al. (2017) Do Correlates of Pain-Related Stoicism and Cautiousness Differ in Younger and Older People with Advanced Cancer? The Journal of Pain 19(3): 301316.

9. Moryl N, Dave V, Glare P, Bokhari A, Malhotra V, et al. (2017) PatientReported Outcomes and Opioid Use by Outpatient Cancer Patients. The Journal of Pain 19(3): 278-290.

10. Inna Belfer, Kristin L Schreiber, John R Shaffer, Helen Shnol, Kayleigh Blaney, et al. Persistent Postmastectomy Pain in Breast Cancer Survivors: Analysis of Clinical, Demographic, and Psychosocial Factors. The Journal of Pain 14(10): 1185-1195.

11. Raymond C. Tait, Kim Zoberi, McKenzie Ferguson, Kimberly Levenhagen, Rebecca A Luebbert, et al. (2018) Persistent Post-Mastectomy Pain: Risk Factors and Current Approaches to Treatment. The Journal of Pain 19(2): 1367-1383.

12. Stephens K, Cooper B, West C, Paul S, Baggott C, et al. (2014) Associations Between Cytokine Gene Variations and Severe Persistent Breast Pain in Women Following Breast Cancer Surgery. The Journal of Pain 15(2): 169-180.

13. McCann B, Miaskowski C, Koetters T, Baggott C, West C, et al. (2012) Associations Between Pro- and Anti-Inflammatory Cytokine Genes and Breast Pain in Women Prior to Breast Cancer Surgery. The Journal of Pain 13(5): 425-437.

14. Miaskowski C, Cooper B, Paul SM, West C, Langford D, et al. (2012) Identification of patient subgroups and risk factors for persistent breast pain following breast cancer surgery. J Pain 13(12):1172-1187.

15. Langford D, Paul S, West C, Abrams G, Elboim C, et al. (2014) Persistent Arm Pain Is Distinct From Persistent Breast Pain Following Breast Cancer Surgery .The Journal of Pain 15(12): 1238-1247D0I: https://doi. org/10.1016/j.jpain.2018.06.002.
(C) (P) This work is licensed under Creative

To Submit Your Article Click Here: Submit Article

DOI: $10.32474 /$ SCSOAJ.2019.02.000134

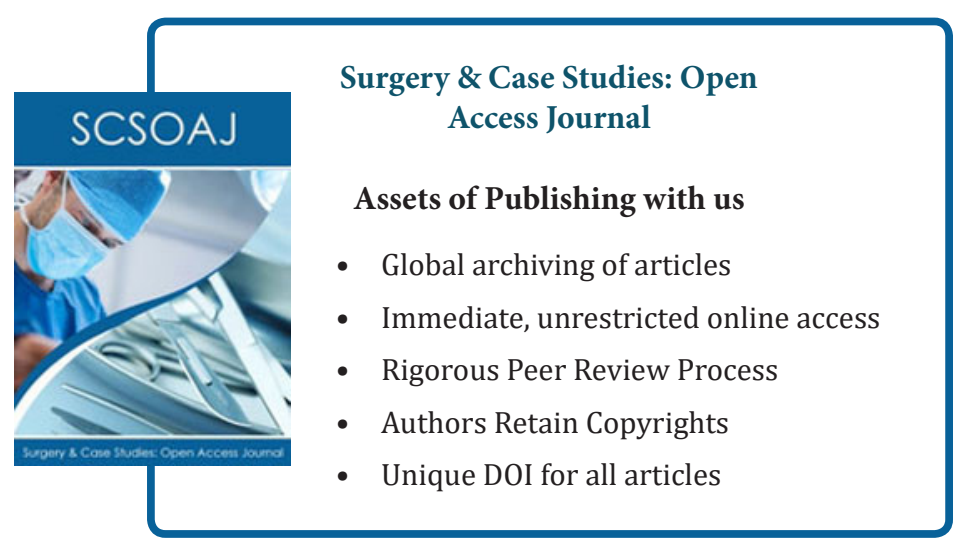

